\title{
Bildung: Kontinuität und Wandel in der Politik der Großen Koalition (2013-2017)
}

Marius R. Busemeyer

\section{Zusammenfassung}

Dieses Kapitel untersucht die Dynamik deutscher Bildungspolitik in der Politik der zweiten Großen Koalition unter Führung Merkels. Üblicherweise wäre im Bildungsföderalismus deutscher Prägung wenig Veränderung zu erwarten. In der Hochschulpolitik hat die Große Koalition jedoch signifikante Reformen auf den Weg gebracht, die nicht mehr ohne weiteres als "Politik der kleinen Schritte“ bezeichnet werden können. Dies gilt insbesondere für die Abschaffung des sogenannten Kooperationsverbots und die Übertragung des BAföG in die alleinige Finanzierungsverantwortung des Bundes. Im Gegensatz dazu zeichnet sich der Bereich der beruflichen Bildung durch ein hohes Maß an politisch gewollter Kontinuität aus, während das Bild des inkrementellen, aber transformativen Wandels besonders gut zur Beschreibung der Entwicklung in der frühkindlichen Erziehung passt. Zusammen genommen dürften jedoch diese Entwicklungen langfristig dazu beitragen, dass sich Deutschland trotz seines institutionellen Erbes als „konservativer Wohlfahrtsstaat“ zunehmend in Richtung eines auf soziale Investitionen ausgerichteten Modells bewegt. 


\section{$1 \quad$ Einleitung}

Im Zuge allgegenwärtiger Debatten zur Zukunft des Sozialstaates im Zeitalter der Digitalisierung und Automatisierung spielt Bildungspolitik eine zentrale Rolle. Der Ausbau von Beteiligungsmöglichkeiten in verschiedenen Bildungsbereichen von der frühkindlichen Erziehung über die schulische und berufliche Bildung bis hin zu den Hochschulen soll und kann dazu beitragen, die Ausprägung von sozialen und bildungsbezogenen Ungleichheiten zu vermindern (Busemeyer 2015b, Solga 2014). Aus diesen Gründen ist Bildungspolitik in den letzten Jahren immer stärker zu einem „Querschnittspolitikfeld“ (Welsh 2015: 469) geworden, in dem das Kerngeschäft der Vermittlung von Bildung und Erziehung durch arbeitsmarkt-, familien- und sozialpolitische Belange ergänzt wird. Dieser Bedeutungszuwachs des Politikfeldes Bildung geht einher mit einem Anstieg der politischen Erwartungen an die Leistungsfähigkeit des Bildungssystems, was dann zu Konflikten führen kann, wenn die Steuerungsfähigkeit des Systems den gestiegenen Ansprüchen nicht gerecht wird.

Wie weiter unten ausführlicher diskutiert wird, steht gerade das Politikfeld Bildung in ganz besonderer Weise im Spannungsfeld zwischen „Parteienwettbewerb und Bundesstaat“ (Lehmbruch 2000), denn hier kollidieren regelmäßig parteipolitisch motivierte Gestaltungsansprüche mit institutionell bedingten Bremsdynamiken. Daher ist es nicht verwunderlich, wenn bisherige Bilanzen der bildungspolitischen Performanz deutscher Bundesregierungen vornehmlich ein hohes Maß an „Kontinuität“ (Welsh 2015: 470) konstatieren. Mahner und Wolf (2010: 399) halten in ihrer Bilanz der Bildungspolitik der ersten Großen Koalition unter Merkel (2005-2009) fest: „Die Erfolge bestehen [...] weniger in spektakulären Einzelbeschlüssen als in einer größeren Zahl von kleineren Maßnahmen [...]. Das Aggregat dieser kleinen Schritte stellt allerdings, insbesondere bei der Hochschulfinanzierung, durchaus einen beachtlichen Wandel dar.“ Dieses Muster des Policy-Wandels erinnert an Streecks und Thelens (2005) Konzept des graduellen, jedoch transformativen Wandels, insbesondere die Überlegung, dass auch scheinbar inkrementelle Veränderungen langfristig transformative Konsequenzen haben können. Der schwarz-gelben Koalitionsregierung (2009-2013) bescheinigt Welsh außerdem ein hohes Maß an „Sachbezogenheit“ (Welsh 2015: 473). Parteipolitisch motivierte Auseinandersetzungen, die in der Geschichte der deutschen Bildungspolitik durchaus vorkamen (Busemeyer 2009b, 2015b, Hepp 2011, Helbig/Nikolai 2015), träten damit zunehmend in den Hintergrund.

Frühere Befunde zur deutschen Bildungspolitik im internationalen Vergleich sind wesentlich kritischer. Bekanntermaßen löste das schlechte Abschneiden Deutschlands bei der ersten PISA-Studie im Jahr 2000 Schockwellen aus, die in 
der Folgeperiode eine Reihe von bildungspolitischen Reformprozessen anstießen (Niemann 2010). Auch wenn Bildungsausgaben nicht zwangsläufig mit Bildungsleistungen korrelieren (Castles 2013) wies Schmidt (2002) bereits frühzeitig darauf hin, dass die Bildungsausgaben in Deutschland im internationalen Vergleich bestenfalls „Mittelmaß“ seien - im Hochschulbereich sogar „noch nicht einmal Mittelmaß“ (Schmidt 2007b). Somit war eine zentrale bildungspolitische Herausforderung der letzten Jahre, diesen Investitionsstau zu beheben und Beteiligungsmöglichkeiten besonders in den auch aus arbeitsmarkt- und sozialpolitischer Perspektive zentralen Bereichen der frühkindlichen Erziehung und der Hochschulbildung auszubauen.

Dieses Kapitel wird untersuchen, ob sich diese grundlegenden Dynamiken deutscher Bildungspolitik auch in der Politik der zweiten Großen Koalition unter Führung Merkels zeigen lassen. Im Bereich der Hochschulpolitik sind allerdings Veränderungen zu beobachten, die nicht mehr ohne weiteres als „Politik der kleinen Schritte" bezeichnet werden können, insbesondere die Abschaffung des sogenannten Kooperationsverbots und die Übertragung des BAföG in die alleinige Finanzierungsverantwortung des Bundes. Im Gegensatz dazu zeichnet sich der Bereich der beruflichen Bildung durch ein hohes Maß an politisch gewollter Kontinuität aus, während das Bild des inkrementellen, aber transformativen Wandels besonders gut zur Beschreibung der Entwicklung in der frühkindlichen Erziehung passt. Zusammen genommen dürften jedoch diese Entwicklungen langfristig dazu beitragen, dass sich Deutschland trotz seines institutionellen Erbes als „konservativer Wohlfahrtsstaat" zunehmend in Richtung eines auf soziale Investitionen ausgerichteten Modells bewegt (Fleckenstein et al. 2011).

\section{$2 \quad$ Bildungspolitik in Deutschland}

Bildungspolitik in Deutschland steht in besonderer Weise und stärker als andere Politikfelder im Spannungsfeld zwischen Parteienwettbewerb und Föderalismusprinzip (Lehmbruch 2000). Die politikwissenschaftliche Bildungsforschung hat inzwischen deutliche Belege dafür, dass Parteipolitik einen großen Einfluss auf die institutionelle Ausgestaltung von Bildungssystemen hat, insbesondere in der historisch wichtigen Phase bildungspolitischer Weichenstellungen in der zweiten Hälfte des 20. Jahrhunderts (Schmidt 1980, 2007a, Castles 1989, Wolf 2006, Rauh et al. 2011, Busemeyer 2007, 2015b, Busemeyer/Seitzl 2017). Auch in Deutschland lassen sich in der Periode nach dem Zweiten Weltkrieg bis in die politisch aufgeladenen späten 1970er Jahre starke ideologische Konflikte in der Bildungspolitik beobachten (Helbig/Nikolai 2015, Hepp 2011, Wolf 2006, Schmidt 1980). Hierbei ging es um 
Fragen zur Schulstruktur (gegliedertes Schulwesen vs. Gesamtschulprinzip), zur Finanzierung (öffentlich vs. privat), die Öffnung des Zugangs zu höherer Bildung sowie die Verbesserung der Durchlässigkeit zwischen unterschiedlichen Sektoren des Bildungssystems, die Beteiligung der Arbeitgeber und anderer privater Akteure an der Bereitstellung von Bildung und den allgemeinen Ausbau von Beteiligungsmöglichkeiten. Unterschiedliche politische Machtverhältnisse in den Bundesländern trugen dazu bei, dass zu Anfang der 1980er Jahre signifikante und systematische Unterschiede in der Bildungspolitik bestanden (Schmidt 1980).

Die föderale Struktur der Bundesrepublik Deutschland müsste diese Unterschiede nicht nur tolerieren, sondern im Geiste des "Wettbewerbsföderalismus" sogar befördern. Gerade im Bereich Bildung zeigt sich jedoch die Wirkmächtigkeit des verfassungsrechtlich kodifizierten Ziels der Herstellung "gleichwertiger Lebensverhältnisse" (Art. 72 Abs. 2 GG). Da die Bildungspolitik auch historisch bedingt vornehmlich in den Zuständigkeitsbereich der Länder fällt, kommt dem Bund traditionellerweise in der Bildungspolitik nur eine recht marginale Rolle zu. Die Angleichung bildungspolitischer Verhältnisse über Bundesländer hinweg geschieht daher vor allen Dingen über den Mechanismus der „horizontalen Koordinierung“, die formal freiwillig erfolgt, de facto aber ein hohes Maß an Verbindlichkeit über die Beschlüsse der Kultusministerkonferenz (KMK) erzeugt (Wolf 2006: 222f.). Die stark ausgeprägte horizontale Koordinierung zwischen Bundesländern schränkt zwar einerseits den Spielraum für parteipolitisch motivierte unterschiedliche Schwerpunktsetzungen ein, kann jedoch nicht verhindern, dass - insbesondere im Hinblick auf Schulstrukturen - inzwischen ein regelrechter „Flickenteppich“ (Welsh 2015: 484) entstanden ist, da neben den klassischen Schultypen Hauptschule, Realschule und Gymnasium eine Reihe von neuen Schulformen entstanden ist (vgl. Helbig/Nikolai 2015 für einen umfassenden Überblick).

Das Verhältnis zwischen Bund und Länderregierungen durchlief verschiedene Entwicklungsphasen - nicht zuletzt auch in Abhängigkeit der parteipolitischen Färbung der jeweiligen Bundesregierung. Die erste Große Koalition (1966-1969) hatte mit einer Änderung des Grundgesetzes zur Einrichtung der Gemeinschaftsaufgabe des Neubaus und Erhalts von Hochschulen den Grundstein für die Bund-Länder-Kooperation im Bereich des Hochschulbaus gelegt, um die besonderen Herausforderungen der rapiden Hochschulexpansion bewältigen zu können. Die Planungseuphorie der sozialdemokratisch-liberalen Koalition der 1970er Jahre führte zur Gründung der Bund-Länder-Kommission für Bildungsplanung und Forschungsförderung, die die Bund-Länder-Kooperation weiter vertiefen sollte. Die christdemokratisch geführten Regierungen der 1980er und 1990er Jahre waren weniger an zentraler Steuerung durch den Bund interessiert. Die rot-grüne Bundesregierung setzte in stärkerer Weise als ihre Vorgängerin auf eine „Politik 
der goldenen Zügel“, indem sie durch Bundeszuschüsse schul- und bildungspolitische Anstöße gab, insbesondere bei dem Investitionsprogramm zum Ausbau der Ganztagsschulbetreuung (siehe unten).

Schließlich beschloss die zweite Große Koalition 2006 im Zuge der großen Föderalismusreform die inzwischen weit vorangeschrittene Politikverflechtung zwischen Bund und Ländern im Bildungsbereich weitestgehend aufzulösen, indem die dominierende Rolle der Länder in der Schulpolitik weiter gestärkt und die verbleibenden Kompetenzen des Bundes in der Hochschulpolitik ganz überwiegend an die Länder delegiert wurden (Mahner/Wolf 2010: 381f.). Dem Bund verblieben lediglich geringe Kompetenzen im Bereich der Forschungsförderung, bei der Regulierung von Hochschulzulassungen und -abschlüssen sowie bei der internationalen Kooperation, wobei der Bund bei letzterem durchaus eine wichtige „Scharnierstellung" übernehmen kann (ebd.: 383).

Dieser kurze Abriss der Entwicklung der Bund-Länder-Beziehungen zeigt zweierlei: Einerseits bestehen durchaus signifikante Unterschiede zwischen den beiden großen Parteien bzw. Parteifamilien hinsichtlich der Ausgestaltung der Rolle des Bundes in der Bildungspolitik: Die SPD setzt sich tendenziell für eine stärkere Zentralisierung bildungspolitischer Verantwortung ein, weil dadurch bestehende Ungleichheiten zwischen Regionen besser ausgeglichen werden könnten. CDU und CSU hingegen treten stärker für eine Bewahrung der Autonomie der Bundesländer ein. Trotz dieser Unterschiede fällt auf, dass wesentliche Weichenstellungen im Bund-Länder Verhältnis in der Bildungspolitik in der Regel von beiden Parteien gemeinsam getroffen wurden, und zwar nicht nur in Form informeller ad-hoc-Kooperation in Deutschlands "Grand Coalition State“ (Schmidt 2008), sondern als Beschlüsse von „echten“ Großen Koalitionen. Diese parteipolitischen Spannungslinien sind durchaus auch in der zweiten Großen Koalition unter Merkels Führung erkennbar.

Abschließend sei noch anzumerken, dass die Komplexität des Politikfeldes Bildung auch deswegen besonders hoch ist, weil sich die Governance-Arrangements in den einzelnen Sektoren des Bildungssystems teilweise deutlich unterscheiden, was mit unterschiedlichen Akteurskonstellationen, Koalitionsdynamiken und Kompetenzverteilungen einhergeht. So hat der Bund im Bereich der Hochschul- und der Berufsbildungspolitik mehr Kompetenzen als in der Schulpolitik, die fest in den Händen der Länder liegt. In der Berufsbildungspolitik sind neben dem Bundesministerium für Bildung und Forschung (BMBF) auch andere Ministerien an der Neuordnung von Ausbildungsberufen beteiligt, vor allen Dingen das Bundesministerium für Arbeit und Soziales (BMAS). In den komplexen Aushandlungsprozessen zur Reform von Ausbildungsberufen sind sowohl die Bundesländer, die für den schulischen Teil der Berufsbildung zuständig sind, als auch Gewerkschaften, Arbeit- 
geberverbände, Kammern und andere Fachverbände involviert. In der Hochschulpolitik hingegen sind die Bundes-Kompetenzen klar auf das BMBF konzentriert, dafür treten allerdings andere Akteure wie der Wissenschaftsrat, die Gemeinsame Wissenschaftskonferenz (GWK), die Universitäten (Hochschulrektorenkonferenz - HRK) und die außeruniversitären Forschungseinrichtungen auf den Spielplan. Auch wenn der Bund in der Schulpolitik kaum formale Gesetzgebungskompetenzen hat, gibt es dennoch immer wieder Konflikte mit den Bundesländern. Der Bereich der frühkindlichen Erziehung wiederum fällt eher in den Kompetenzbereich des Bundesministeriums für Familien, Senioren, Frauen und Jugend (BMFSFJ). Da es hier um familienpolitische Kompetenzen geht, hat der Bund ein größeres Mitsprachrecht als in der Schulpolitik, was letztlich auch eine wesentliche Triebkraft hinter der jüngsten Expansion dieses Bildungsbereiches war.

Kurzum: Es gibt wohl kaum ein anderes Politikfeld, in dem unterschiedliche politische Arenen in derartiger Weise miteinander verschränkt sind wie in der Bildungspolitik: Neben die föderale Konfliktlinie treten parteipolitische Spannungen sowie Interessengegensätze zwischen Arbeitgebern und Gewerkschaften. Hinzu kommen Kompetenz- und Abgrenzungskonflikte zwischen unterschiedlichen Bundesministerien. Diese Konstellation lässt erwarten, dass trotz hohen Problemdrucks und einer ausgeprägten öffentlichen Nachfrage nach Bildungsreformen eher inkrementelle Veränderungen zu erwarten sind.

\section{$3 \quad$ Positionen der Parteien bei Regierungsantritt}

Bevor die Reformen der zweiten Großen Koalition unter Merkels Führung analysiert werden, soll zunächst ein Blick in die Wahlprogramme und den Koalitionsvertrag gewagt werden. Generell ist hierbei zu bedenken, dass Bildung im Parteienwettbewerb einen hohen Stellenwert hat. Eine repräsentative Umfrage zur öffentlichen Meinung im Bereich Bildungspolitik aus dem Jahr 2014 zeigt, dass sich mehr als 80 Prozent der befragten Deutschen für mehr oder sogar viel mehr öffentliche Ausgaben im Bildungsbereich einsetzen (Busemeyer et al. 2018: 41). Der Stellenwert der Bildung im Vergleich zu anderen Sozialpolitiken wie Rente, Arbeitslosen- oder Krankenversicherung ist hierzulande besonders hoch: Wenn sich die Befragten für ein Politikfeld entscheiden müssten, in dem der Staat mehr ausgibt, dann sprechen sich 40 Prozent für den Bereich Bildung aus. Familienpolitik (das am zweithäufigsten genannte Politikfeld) erreicht lediglich 19 Prozent Zustimmung (ebd.: 10). Vor diesem Hintergrund ist es nicht verwunderlich, dass Parteien unterschiedlichster Couleur in Wahlkämpfen die Förderung von Bildung 
fordern. Forderungen nach einem gezielten Rückbau von Bildung kommen so gut wie nie vor (Jakobi 2011), allerdings unterscheiden sich Parteien deutlich darin, wie stark sie Bildung als Politikziel betonen (Busemeyer et al. 2013). Es geht also nicht nur darum, welche Positionen einzelne Parteien in der Bildungspolitik einnehmen, sondern ganz zentral auch um die Frage, wie hoch die Salienz des Themas für verschiedene Parteien ist.

Die Lektüre der Wahlprogramme der großen Parteien zur Bundestagswahl 2013 zeigt allerdings erstaunlich große Überschneidungen, und zwar sowohl hinsichtlich der Positionen, als auch bei der Salienz von Bildungspolitik. In beiden Programmen werden zunächst grundsätzliche Problemstellungen in der Wirtschafts- und Finanzpolitik angesprochen; direkt im Anschluss werden allerdings bildungspolitische Ziele vorgestellt. Obwohl es sich um Programme für die Bundestagswahl handelt, werden in beiden Programmen auch Fragen zur Schulpolitik angesprochen, auch wenn dies eigentlich Ländersache ist (CDU/CSU 2013: 21ff., SPD 2013: 43ff.). Konkrete inhaltliche Gemeinsamkeiten zeigen sich bei den genannten Themen: Förderung der frühkindlichen Erziehung, Ausbau der Ganztagsbetreuung in Schulen, Verbesserung der Qualität der Lehrer*innen-Ausbildung, Erhalt und Ausbau der Stärken der dualen Berufsausbildung, Förderung der Kooperation zwischen Bund und Ländern und Umsetzung der nationalen Bildungsstandards, Ausbau der Studienfinanzierung (BAföG) und Verbesserung der finanziellen und strukturellen Rahmenbedingungen an Hochschulen. Hierbei ist auffallend, dass vor allen Dingen die CDU/CSU klassisch sozialdemokratische Themen übernommen hat.

Natürlich finden sich bei genauerer Betrachtung auch einige Unterschiede in der Akzentsetzung. Die CDU/CSU bezeichnet die „rot-grüne Einheitsschule“ als "Irrweg" und spricht sich klar für den Erhalt des Gymnasiums aus (CDU/CSU 2013: 21), allerdings auch nicht deutlich gegen die neuen Varianten von teilintegrativen Schulen, die inzwischen auf Landesebene als Alternative zum Gymnasium entstanden sind (wie etwa die Gemeinschaftsschulen in Baden-Württemberg oder die Realschule Plus in Rheinland-Pfalz). Die Unionsparteien sprechen sich außerdem für die Förderung von Schulen in freier oder privater Trägerschaft sowie die feste Verankerung von Religionsunterricht als Schulfach aus (ebd.: 22). Zwar fordern auch CDU und CSU „[m]ehr Zusammenarbeit zwischen Bund und Ländern“ (ebd.: 21), setzen sich aber klar für eine Beibehaltung der getrennten Zuständigkeiten zwischen Bund und Ländern ein. Die SPD geht hier weiter, denn sie verlangt explizit die Aufhebung des Kooperationsverbots in der Bildungspolitik (SPD 2013: 44f.). Sie befürwortet außerdem die Abschaffung des Betreuungsgeldes - ein Prestigeprojekt der CSU in der schwarz-gelben Regierungskoalition (vgl. Henninger/von Wahl 2015).

Auch die Wahlprogramme der kleineren Parteien weisen bei einigen zentralen Fragen hohe Überschneidungen mit der Programmatik der größeren Parteien auf, 
insbesondere bei Forderungen nach der Anhebung der Bildungsinvestitionen mit einem Schwerpunkt im Hochschulbereich, dem Ausbau von frühkindlicher Erziehung und Ganztagsbetreuung sowie des BAföG. Kleinere Parteien setzen allerdings auch eigene Akzente. So spricht sich die FDP noch deutlicher als die CDU/CSU für „Freiheit, Vielfalt und Leistungswettbewerb“ im „Bildungsföderalismus“ (FDP 2013: 26), die Stärkung der Autonomie von Schulen und Hochschulen (ebd.: 26, 28) sowie die Fortführung der Exzellenzinitiative in der Forschungsförderung aus (ebd.: 29). Für die Grünen haben die Themen Inklusion in der Bildung, Stärkung der partizipativen Strukturen in Schulen und Universitäten sowie Beseitigung von Bildungsungleichheiten durch Ausweitung des Zugangs zu verschiedenen Bildungsstufen einen besonders hohen Stellenwert (Bündnis 90/Die Grünen 2013: 104-109). Auch für die Linke ist das Thema freier Zugang zu Bildung zentral (Die Linke 2013: 33). Sie positioniert sich außerdem gegen die Beteiligung von privaten Akteuren in der Bereitstellung und Finanzierung von Bildung (ebd.: 33) und befürwortet in klaren Worten die Überwindung des gegliederten Schulsystems und die Einführung einer „Gemeinschaftsschule als Regelschule, in der alle Kinder und Jugendlichen länger gemeinsam lernen“ (ebd.: 34). Sowohl Grüne als auch Linke treten für die Abschaffung des Kooperationsverbots in der Bildungspolitik ein (Bündnis 90/Die Grünen 2013: 106, Die Linke 2013: 34).

In der Gesamtschau zeichnet sich somit zwischen den Parteien ein vergleichsweise großer Konsensbereich ab, der von der Akzentsetzung der kleineren Parteien an den Rändern flankiert wird. Der Koalitionsvertrag zwischen CDU, CSU und SPD deckt diesen Konsensbereich in weiten Teilen ab (CDU/CSU/SPD 2013). Allerdings sind die Inhalte des Koalitionsvertrags im Vergleich zu den Wahlprogrammen der Parteien sehr viel stärker auf die tatsächlichen Kompetenzen des Bundes in der Bildungspolitik zugeschnitten, die schwerpunktmäßig in der Förderung von Wissenschaft und Forschung, der Berufsbildungspolitik sowie - unter familienpolitischen Vorzeichen - der frühkindlichen Erziehung liegen. Allgemeine Fragen zur Bildungs- und Schulpolitik, die eher in den Kompetenzbereich der Länder fallen und zudem ideologisch umstrittener sind, werden kaum angesprochen. Des Weiteren fällt auf, dass der Koalitionsvertrag in weiten Teilen auf bestehenden Initiativen und Politikansätzen aufbaut und diese fortführen will, beispielsweise den Hochschulpakt, die Exzellenzinitiative und den Pakt für Forschung und Innovation, der vornehmlich die außeruniversitären Forschungseinrichtungen betrifft (ebd.: 20). Das strittige Thema des Kooperationsverbots wird im Koalitionsvertrag nicht angesprochen, auch wenn mehr Bundesmittel zur Verbesserung der Grundfinanzierung der Hochschulen in Aussicht gestellt werden (ebd.: 21). Die SPD hatte sich in den Koalitionsverhandlungen getreu ihres Wahlprogrammes für die Abschaffung des Kooperationsverbotes eingesetzt, und zwar auch in 
den Bereichen, die nicht direkt die Hochschulen betreffen. Sie konnte sich damit aber nicht gegen die Unionsparteien, vor allen Dingen den Widerstand der CSU, durchsetzen (Spiewak 2013).

Auch im Bereich der beruflichen Bildung setzt die Große Koalition auf die Fortführung und Weiterentwicklung von bestehenden Strukturen statt auf radikale Veränderungen. Der 2004 erstmals zwischen Regierung und Arbeitgebern geschlossene und seitdem regelmäßig erneuerte Ausbildungspakt soll in Kooperation mit den Ländern und den Sozialpartnern zu einer „Allianz für Aus- und Weiterbildung" weiterentwickelt werden (CDU/CSU/SPD 2013: 23). Die dezidierten Hinweise auf „die Sozialpartner“ und die Bedeutung des „Konsensprinzips in der Berufsordnungsarbeit" (ebd.: 24) deuten an, dass die Bundesregierung eine explizite Aufwertung der Rolle der Gewerkschaften anstrebt. Die rot-grüne Regierung hatte 2004 - zum Beispiel im Rahmen der ersten Auflage des Ausbildungspaktes oder bei der Neuordnung von zweijährigen Ausbildungsberufen - die Gewerkschaften noch eher stiefmütterlich behandelt (Busemeyer 2012).

Der Bereich der frühkindlichen Erziehung wird recht knapp und im Kontext weiterer familienpolitischer Maßnahmen ohne direkten Bezug zur Bildungspolitik abgehandelt (CDU/CSU/SPD 2013: 69). Die Koalition nimmt sich vor, die Qualität der Kinderbetreuung „weiter voranzutreiben“ (ohne allerdings konkrete Qualitätsziele wie etwa Betreuungsschlüssel zu nennen) und die „Ganztagsbetreuung in Kindertageseinrichtungen" auszubauen. Der Ausbau von Ganztagsschulen wird allerdings weder hier noch im Bildungskapitel ausführlich erwähnt.

Alles in allem suggeriert der Koalitionsvertrag somit keinen radikalen Neuanfang in der Bildungspolitik, sondern eher eine bestenfalls etwas entschiedenere Fortsetzung und Weiterentwicklung von Strukturen und Initiativen, die sich bewährt haben oder von den relevanten Akteuren zumindest so wahrgenommen wurden. Themen, die zwischen den großen Parteien noch strittig waren, wie etwa das Kooperationsverbot oder die Schulstrukturfrage, wurden ausgeklammert. Mit Johanna Wanka wurde eine Bildungsministerin im Amt bestätigt, die bereits in den letzten Monaten der schwarz-gelben Koalition berufen worden war. Im Vergleich zu ihrer Vorgängerin Annette Schavan ist Wanka weniger Parteipolitikerin, sondern - aufbauend auf ihrer Erfahrung als Wissenschaftsministerin in Brandenburg und Niedersachen sowie als Rektorin und Hochschulprofessorin - stärker Fachpolitikerin. Insofern deuteten die Zeichen zu Anfang der Regierungsperiode der zweiten Großen Koalition unter Merkels Führung sehr stark auf Kontinuität statt Wandel in der Bildungspolitik hin. Dies sollte sich allerdings nur teilweise bestätigen. 


\section{Bilanz der Bildungspolitik der Großen Koalition, 2013-2017}

\subsection{Hochschulpolitik}

Nach der Föderalismusreform von 2006 verblieben dem Bund nur wenige Kompetenzen im Bereich der Hochschulpolitik, insbesondere zur Förderung von außeruniversitärer Forschung und von zeitlich und thematisch begrenzten Projekten im Bereich der Hochschulen. Aus diesem Grund wurde eine Reihe von „Pakten“verabschiedet, die formal Verwaltungsvereinbarungen zwischen Bund und Ländern sind. Diese hatten im Wesentlichen das Ziel, den Bundesländern, deren finanzieller Spielraum durch die im Rahmen der Föderalismusreform 2 beschlossenen Schuldenbremse zunehmend knapper wurde, durch die gezielte Bereitstellung von Bundesmitteln unter die Arme zu greifen. Auch wenn die Pakte grundsätzlich temporär befristet sind, können sie in den betreffenden Zeiträumen durchaus signifikante Mittelzuflüsse an die begünstigten Hochschulen generieren. Zusammen genommen tragen sie de facto zu einer graduellen Transformation der deutschen Hochschulfinanzierung bei: Das Paktinstrument führt dazu, dass sich über die reguläre Drittmittelfinanzierung hinaus das Prinzip der kompetitiven Mitteleinwerbung auch in jenen Bereichen der Hochschulen verbreitet, die eigentlich durch die Grundfinanzierung abgedeckt sein könnten (wie etwa Lehre oder Lehrerausbildung).

Einige Pakte wurden bereits durch Vorgängerregierungen abgeschlossen, aber in der Regierungsverantwortung der Großen Koalition erneuert bzw. verlängert. Hier ist zunächst der Hochschulpakt $2020 \mathrm{zu}$ nennen. Die erste Phase des Hochschulpaktes begann 2006 - ursprünglich mit dem Ziel, zur Bewältigung des erwarteten Anstiegs der Studierendenzahlen, verschärft durch die Problematik der doppelten Abiturjahrgänge nach Einführung von G8 in vielen Bundesländern und der Abschaffung der Wehrpflicht, begrenzt zusätzliche Mittel zur Finanzierung von weiteren Studienplätzen zur Verfügung zu stellen. Tatsächlich ist die Zahl der Studierenden von knapp 2 Mio. im Jahr 2006 auf 2,8 Mio. im Jahr 2016 deutlich angestiegen (Statista 2018). Dementsprechend sind auch die öffentlichen Ausgaben des Bundes und der Länder deutlich von 18,4 Mrd. Euro im Jahr 2005 auf 28,6 Mrd. im Jahr 2015 gewachsen (Statistisches Bundesamt 2016: 66). Da der Anstieg der Studierendenzahlen bereits während der zweiten Phase stärker war als ursprünglich erwartet, wurde die Finanzierung des Hochschulpaktes aufgestockt.

Die dritte - und bis auf weiteres letzte - Phase des Hochschulpaktes wurde Ende 2014 zunächst in der Gemeinsamen Wissenschaftskonferenz (GWK), dann in Form einer gemeinsamen Entscheidung von Bundeskanzlerin und Regierungschefs der Länder eingeleitet. In dieser dritten Phase werden Bund und Länder im Vergleich 
zum Referenzjahr 2005760.033 zusätzliche Studienmöglichkeiten finanzieren (Statistisches Bundesamt 2016: 64). In den Jahren 2015 bis 2018 stellt der Bund 5,37 Mrd. Euro zu Ausfinanzierung der Studienanfänger*innen der zweiten Phase sowie weitere Mittel im Umfang von 8,78 Mrd. Euro zur Finanzierung der Studienanfänger*innen der Paktphase 2016 bis 2020 und deren Ausfinanzierung bis 2023 zur Verfügung. Insgesamt wird der Bund zwischen 2007 und 2020 20,2 Mrd. Euro zur Finanzierung zusätzlicher Studienplätze bereitgestellt haben, verglichen mit einem Beitrag von 18,3 Mrd. Euro von Seiten der Länder (Statistisches Bundesamt 2016: 64). An diesen Zahlen wird deutlich, dass sich der Bund über den Hochschulpakt in wesentlichem Maße an der Finanzierung von universitärer Lehre beteiligt.

$\mathrm{Zu}$ dem quantitativ bedeutsamen Hochschulpakt 2020 kommt der „Pakt für Forschung und Innovation" hinzu, den Bund und Länder mit den außeruniversitären Forschungseinrichtungen geschlossen haben. Dies sind die Deutsche Forschungsgemeinsacht (DFG), die Fraunhofer-Gesellschaft, die Helmholtz-Gemeinschaft, die Max-Planck-Gesellschaft und die Leibniz-Gemeinschaft. Auch hier wurde Ende 2014 eine Entscheidung über die Fortführung des Paktes in den Jahren 2016-2020 getroffen. Die außeruniversitären Forschungseinrichtungen verpflichten sich hierbei auf forschungspolitische Ziele und erhalten im Gegenzug eine Zusage über jährliche Budgetsteigerungen von 3 Prozent - das sind insgesamt 3,9 Mrd. Euro für die neue Paktphase, die vom Bund alleine getragen werden (vgl. BMBF o. J. a).

Weitere, schon durch die Vorgängerregierung beschlossene Pakte sind der „Qualitätspakt Lehre“ und die „Qualitätsoffensive Lehrerbildung“. Für den Qualitätspakt Lehre stellt der Bund zwischen 2011 und 2020 in zwei Förderphasen insgesamt 2 Mrd. Euro zur Verfügung, die zur Verbesserung der Qualität der Lehre und der Betreuung der Studierenden eingesetzt werden. Die Qualitätsoffensive Lehrerbildung fördert mit bis zu 500 Mio. Euro an Bundesmitteln ebenfalls in zwei Förderphasen von 2014 bis 2023 Projekte, die innovative Ansätze zur Verbesserung der Ausbildung von Lehrer*innen umsetzen möchten.

Neben der Weiterführung bestehender Pakte wurde durch eine umfassende Vereinbarung zwischen Bund und Ländern (und zwischen den Koalitionspartnern der Großen Koalition) im Mai 2014 eine umfassende Vereinbarung zur Zukunft der Bildungsfinanzierung getroffen, die langfristig nachhaltige Auswirkungen haben dürfte (BMF 2014). Die Länder waren durch die steigenden Studierendenzahlen sowie den Ausbau der Betreuungsmöglichkeiten in der frühkindlichen Erziehung finanziell immer stärker unter Druck geraten. Selbst in den wohlhabenden Südländern, aber vor allen Dingen in den neuen Bundesländern, gingen die Ausgaben pro Studierendem zwischen 2000 und 2011 teilweise deutlich zurück (Demling 2014). Aus diesem Grund wurde vereinbart, ab 2015 dem Bund die alleinige Finanzierungsverantwortung für das Studien-BAföG zu übertragen; bisher hatten 
die Länder 35 Prozent der Kosten des BAföG finanziert. Dementsprechend wurde in Zukunft mit einer Entlastung von etwa 1,14 Mrd. Euro pro Jahr für die Länder gerechnet (Titz 2014). Die Länder sagten zu, die freiwerdenden Mittel für zusätzliche Ausgaben im „Bereich Hochschule und Schule“ zu verwenden.

Gewissermaßen als Ausgleich für die Übertragung der Finanzierungsverantwortung des BAföG an den Bund wurde außerdem eine Änderung des Art. 91b GG vereinbart, die einer Abschaffung des Kooperationsverbots im Bereich der Hochschulen gleichkam. Einige SPD-Politiker - allen voran Ministerpräsident Stephan Weil aus Niedersachsen - sprachen sich für eine weitergehende Regelung und eine Abschaffung des Kooperationsverbots auch in anderen Bildungsbereichen aus (Himmelrath 2014), konnten sich mit diesen Forderungen allerdings nicht durchsetzen. Wie die kursorische Übersicht der Wahlprogramme im vorangehenden Abschnitt gezeigt hat, war Widerstand in diesem Fall eher von Seiten der Unionsparteien, vor allen Dingen der CSU, zu erwarten. Ministerin Wanka hatte sich allerdings schon früh persönlich für die Abschaffung des Kooperationsverbots ausgesprochen und eingesetzt.

So wurde Ende 2014 in Bundestag und Bundesrat eine Änderung des Art. 91b Abs. 1 GG beschlossen, die zeitgleich zur BAföG-Reform zum 1. Januar 2015 in Kraft trat. Die neue Fassung des Art. 91b Abs. 1 GG erlaubt eine Kooperation von Bund und Ländern ,in Fällen überregionaler Bedeutung bei der Förderung von Wissenschaft, Forschung und Lehre“. Allerdings bedürfen „Vereinbarungen, die im Schwerpunkt Hochschulen betreffen, [...] der Zustimmung aller Länder". Der letztere Passus gibt demnach auch einzelnen Bundesländern explizit ein faktisches Veto-Recht, wenn es um Kooperationen in der Hochschulpolitik im engeren Sinne geht. Im Zuge der BAföG-Reform wurden außerdem die Bedarfssätze und Einkommensfreibeträge der Eltern angehoben, was die Bedingungen der Studienfinanzierung signifikant verbesserte.

Bei der Umsetzung der umfassenden Finanzierungsvereinbarung - insbesondere der Verwendung der auf Länderebene durch die BAföG-Reform freiwerdenden Mittel - zeichneten sich bald Konflikte ab, denn die Zusage der Verwendung dieser Mittel für den Bildungsbereich wurde von den Bundesländern unterschiedlich interpretiert und ausgestaltet (vgl. Schmoll 2015). Einige Bundesländer (Hessen, Bayern und Sachsen) investierten die BAföG-Mittel zu 100 Prozent in Hochschulen, andere (Baden-Württemberg, Rheinland-Pfalz, Mecklenburg-Vorpommern, Brandenburg und Berlin) nur zu 40 bis 60 Prozent. Wiederum andere (Niedersachen, Nordrhein-Westfalen, Hamburg und Schleswig-Holstein) verwendeten die Mittel zur Querfinanzierung von anderen Bereichen des Bildungssystems, wie etwa Schulen oder die frühkindliche Erziehung. Trotz der Proteste von Ministerin Wanka gegen diese Vorgehensweise der Länder waren und sind die Möglichkeiten 
des Bundes begrenzt, die tatsächliche Verwendung der ehemaligen BAföG-Mittel auf Länderebene zu beeinflussen.

Auf der Grundlage der Abschaffung des Kooperationsverbots im Bereich Hochschulen wurden in Folge neue Vereinbarungen zwischen Bund und Ländern zur Bildungsfinanzierung geschlossen, die im Juni 2016 als „Gesamtpaket für die Hochschulen“ von Bund und Ländern verabschiedet wurden (BMBF 2016a). Diese Vereinbarungen haben auf den ersten Blick wieder Pakt-Charakter, können im Unterschied zu den vorherigen allerdings nun prinzipiell ohne zeitliche Beschränkung geschlossen werden. Dieses Modell findet Anwendung bei der Neuauflage der Exzellenzinitiative zur Förderung von Spitzenforschung an Universitäten, die nun folgerichtig als „Exzellenzstrategie“ bezeichnet wird. Ab 2018 stellen Bund und Länder jährlich 533 Mio. Euro bereit, wobei 75 Prozent der Mittel vom Bund getragen werden und 25 Prozent vom Sitzland der jeweiligen Exzellenzeinrichtung.

Die Exzellenzstrategie (kurz: „ExStrat“) knüpft in vielerlei Hinsicht an die vorangegangene Exzellenzinitiative an, insbesondere beim Prinzip der kompetitiven Vergabe von zusätzlichen Forschungsmitteln für Spitzenforschung an Universitäten. Die neue ExStrat umfasst zwei Förderlinien: „Exzellenzcluster“ und „Exzellenzuniversitäten“. Für Exzellenzcluster werden projektbezogen Fördermittel zwischen 3 und 10 Mio. Euro jährlich pro Cluster vergeben; die Laufzeit der Cluster beträgt maximal 14 Jahre (in zwei Förderphasen von jeweils sieben Jahren unterteilt). Exzellenzuniversitäten erhalten dauerhaft zusätzliche Förderung, müssen sich allerdings alle sieben Jahre einer externen Prüfung unterziehen. Antragsstellende Universitäten müssen mindestens zwei Cluster (bzw. drei bei Universitätsverbünden) vorweisen können, um sich auf den Status einer Exzellenzuniversität bewerben zu können. Es ist geplant, etwa 45-50 Cluster und elf Exzellenzuniversitäten einzurichten. Anträge werden im Rahmen eines wissenschaftsorientierten Peer-Review-Prozesses begutachtet. Die endgültige Entscheidung über die Verteilung der Mittel fällt im Herbst 2018.

Die Weiterentwicklung der Exzellenzinitiative war ein wichtiger Bestandteil des Koalitionsvertrags der Großen Koalition (siehe oben). Insofern herrschte ein breiter Konsens hinsichtlich der prinzipiellen Fortführung dieser Art der Forschungsförderung. Gleichwohl gab es in der konkreten Verhandlungsphase durchaus einige Konflikte zwischen den Koalitionspartnern. Die SPD setzte sich tendenziell für eine breitere Verteilung der Exzellenzmittel ein und schlug anfangs die Förderung von regionalen Verbünden statt einzelner Exzellenzuniversitäten vor. Die Union hingegen wollte die Förderung auf möglichst wenige Spitzenuniversitäten konzentrieren (Wiarda 2015). Die abschließende Vereinbarung zur ExStrat spiegelt beide Positionen wider, denn sie ermöglicht und fördert die Kooperation von Universitäten miteinander und mit außeruniversitären Einrichtungen im Rahmen von Exzellenzanträgen, 
erlaubt aber auch die Einzelantragsstellung. Kurz vor Abschluss der Verhandlungen drohte Hamburg zudem mit einem Veto (Spiegel Online 2016). Hier ging es um die Frage, ob nach der Kür der ersten Riege von Exzellenzuniversitäten 2018 sieben Jahren später (also 2025) neue Universitäten in den elitären Kreis aufgenommen werden können sollten. Der gefundene Kompromiss sieht nun vor, dass 2025 bis zu vier weitere Universitäten zu Exzellenzuniversitäten ernannt werden können.

Die ExStrat wird flankiert durch zwei weitere Pakte, die allerdings zeitlich befristet sind. Über das „Programm zur Förderung des wissenschaftlichen Nachwuchses" stellt der Bund zwischen 2017 und 20321 Mrd. Euro zur Schaffung von 1.000 zusätzlichen Tenure-Track-Professuren zur Verfügung. Mit der Initiative „Innovative Hochschule“werden bis 2027 insgesamt 500 Mio. Euro zur Förderung von Wissens- und Technologietransfer ausgegeben, wobei sich dieses Programm vornehmlich an kleine und mittlere Universitäten und Fachhochschulen richtet.

Im März 2016 wurde außerdem eine Reform des Wissenschaftszeitvertragsgesetzes beschlossen (vgl. zum Folgenden BMBF o. J. b), das ursprünglich 2007 in Kraft trat. Das Gesetz beinhaltet Sonderregelungen für befristete Beschäftigungsverhältnisse von Wissenschaftler ${ }^{\star}$ innen an Hochschulen und Forschungseinrichtungen. Das Ziel der Neuregelung ist es, die Beschäftigungssituation des wissenschaftlichen Nachwuchses zu verbessern. Wie im „Bundesbericht Wissenschaftlicher Nachwuchs“ dokumentiert, ist der Anteil befristeter Beschäftigungsverhältnisse an deutschen Hochschulen und Forschungseinrichtungen sehr hoch; zudem sind die Laufzeiten der Arbeitsverträge oftmals sehr kurz, und Promovierende arbeiten häufig mehr als die vertragliche vereinbarte Arbeitszeit (Konsortium 2017: 27). Die Neuregelung des Wissenschaftszeitvertragsgesetzes soll hier durch mehrere Maßnahmen Abhilfe schaffen, wie etwa die Unterbindung von Kurzbefristungen, indem Vertragslaufzeiten grundsätzlich so bemessen werden, dass sie der Dauer der zu erreichenden wissenschaftlichen Qualifizierung entsprechen. Bei Drittmittelprojekten sollen sich die Vertragslaufzeiten an den Projektlaufzeiten orientieren. Nicht-wissenschaftliches Personal, das in einem drittmittelfinanzierten Projekt arbeitet, kann zukünftig nicht mehr grundsätzlich auf Grundlage des Wissenschaftszeitvertragsgesetzes befristet beschäftigt werden.

Innerhalb der Großen Koalition war die Neuregelung des Gesetzes vergleichsweise wenig umstritten; es gab allerdings deutliche Kritik von Seiten der Wissenschaft einerseits und von der Bildungsgewerkschaft GEW sowie den Oppositionsparteien andererseits. In einem Brief an Bildungsministerin Wanka kritisierte eine Allianz von Wissenschaftsorganisationen im Juni 2015 die Pläne der Regierung als zu weitreichende Beschneidung der Flexibilität der Hochschulen und Forschungseinrichtungen (Kramer 2015). Flexibilität bei der Befristung von Arbeitsverträgen - auch von nicht-wissenschaftlichem Personal - seit notwendig, um Spitzenforscher*innen 
attraktive Arbeitsbedingungen bieten zu können. Die GEW sowie die Grünen und die Linke als Oppositionsparteien hingegen kritisierten das Gesetz als zu unpräzise und abstrakt, so dass bestehende Defizite in der Beschäftigungspraxis damit nicht effektiv beseitigt werden könnten (Spiegel Online 2017).

\subsection{Berufliche Bildung}

Im Bereich der beruflichen Bildung deutet die Sprache des Koalitionsvertrages eher auf inkrementelle Anpassungen statt radikale Veränderungen hin. Das deutsche Berufsbildungswesen wird als „Erfolgsmodell“ bezeichnet (CDU/CSU/ SPD 2013: 23). Es soll daher nicht grundsätzlich reformiert werden, sondern die Koalitionspartner wollen die duale Ausbildung - das Herzstück des deutschen Berufsbildungssystems - „stärken und modernisieren“ (ebd.: 24). Eine konkrete Vorgabe des Koalitionsvertrags in dieser Hinsicht ist eine Evaluation der letzten Reform des Berufsbildungsgesetzes aus dem Jahr 2005. Diese Reform hatte unter anderem das Modell der gestuften Ausbildungsgänge wiedereingeführt und dadurch die Differenzierung des Systems beschleunigt, die Internationalisierung der Berufsbildung vorangetrieben und zur Bewältigung von kurzfristigen Engpässen auf dem Ausbildungsmarkt die Möglichkeit geschaffen, neben regulären betrieblichen Ausbildungen auch vollzeitschulische Ausbildungsgänge in dualen Ausbildungsberufen einzurichten (vgl. Busemeyer 2009b: 157ff.).

Im März 2016 legte das Bundesbildungsministerium den angekündigten Evaluierungsbericht vor (BMBF 2016b). Der Grundtenor dieses Berichtes deutete sehr stark auf Kontinuität statt Wandel in der beruflichen Bildung hin. Die Novelle des Berufsbildungsgesetzes (BBiG) habe sich „bewährt“ und sei in der Praxis „sehr gut" aufgenommen worden; ein grundsätzlicher Reformbedarf werde daher nicht gesehen (ebd.: 4). Dementsprechend schlägt der Bericht „eher technische[...] und detailoptimierende[...]“ Anpassungen des BBiG vor (ebd.: 5f.). Zudem empfiehlt der Bericht eine vorsichtige Ergänzung des BBiG im Hinblick auf eine bessere rechtliche Verankerung des Kompetenzbegriffs, so dass eine bessere Kompatibilität zwischen den BBiG-Regelungen und dem kompetenzorientierten Ansatz des Deutschen Qualifikationsrahmens (DQR) erreicht werden könne.

Auch wenn die Gewerkschaften das Modell der dualen Ausbildung generell wertschätzen und unterstützen, äußerten sie doch verhaltene Kritik an dem Evaluationsbericht des BMBF. Statt die Evaluation des BBiG an eine externe Forschungseinrichtung zu geben, wurde sie vom BMBF in Kooperation mit dem Bundesinstitut für Berufsbildung (BIBB) durchgeführt und stelle daher eher eine „politische und juristische Bewertung der Funktionsfähigkeit des BBiG“ dar statt 
einer umfassenden Evaluation (DGB 2016: 3). Aus gewerkschaftlicher Sicht weiterhin bestehende Probleme - wie etwa Qualitätsprobleme in manchen Ausbildungsberufen, schwierige Übergänge für Jugendliche mit geringen Schulqualifikationen oder die zurückgehende Ausbildungsbereitschaft von Betrieben - seien von „vornherein ausgeklammert“ oder gar „verschwiegen“ worden (DGB 2016: 3). Die Arbeitgeber hingegen teilen die generell positive Einschätzung des BMBF und sehen ebenfalls keinen grundsätzlichen Reformbedarf (Deutscher Bundestag 2017).

Analog zur Anhebung der BAföG-Sätze (siehe oben) wurden zum 1. August 2016 ebenfalls die Fördersätze für Leistungen nach dem Aufstiegsfortbildungsfördergesetz (AFBG, auch „Meister-Bafög“ genannt) verbessert (Bundesregierung 2016). Im Rahmen dieses Gesetzes werden gezielt Personen gefördert, die eine Bildungskarriere im beruflichen Aus- und Fortbildungssystem verfolgen. Im Jahr 2014 waren dies 171.000 Geförderte. Die Gesetzesnovelle verbessert - analog zur BAföG-Novelle - die Fördersätze und Einkommensfreibeträge sowie die Bonuszahlungen für „Meisterstücke“ und erfolgreiche Abschlussprüfungen. Insgesamt stellen Bund und Länder in den vier Jahren nach Inkrafttreten des Gesetzes 250 Mio. Euro zusätzlich für die Aufstiegsfortbildung zur Verfügung.

Der Koalitionsvertrag sieht außerdem die Weiterentwicklung des Ausbildungspaktes zu einer "Allianz für Aus- und Weiterbildung“ vor (CDU/CSU/SPD 2013: 23). Der erste Ausbildungspakt (der „Nationale Pakt für Ausbildung und Fachkräftenachwuchs in Deutschland") wurde 2004 zwischen der Bunderegierung und den Wirtschaftsverbänden geschlossen (DIHK, BDA, ZDH und BDI). Die damalige rot-grüne Regierung hatte zuvor einen Gesetzesentwurf zur Einführung einer Ausbildungsumlage vorgelegt, um die Wirtschaft angesichts fehlender Ausbildungsstellen dazu zu bewegen, mehr Ausbildungsplätze anzubieten (vgl. zum Folgenden Busemeyer 2009b: 151ff.). Nicht zuletzt war der Vorschlag einer Ausbildungsumlage auch innerhalb des damaligen Regierungslagers umstritten, so dass der Vorschlag zur Verabschiedung eines Paktes letztlich von Arbeitgeberseite als Kompromiss angenommen wurde. In dem Pakt sagten die Arbeitgeber zu, jährlich 30.000 neue Ausbildungsplätze einzuwerben sowie 25.000 Plätze für betriebliche Einstiegsqualifikationen anzubieten. Von Gewerkschaftsseite wurde kritisiert, dass der Pakt nicht verbindlich vorschrieb, dass die neuen Ausbildungsplätze auch tatsächlich zusätzliche Ausbildungsplätze sein müssen. Auch aus diesem Grund beteiligten sich die Gewerkschaften somit nicht am Ausbildungspakt - nicht zuletzt wurde befürchtet, dass der Pakt die bestehenden korporatistischen Entscheidungsstrukturen unterminieren könnte.

Der Ausbildungspakt wurde in der Folge zweimal neu aufgelegt (2007 und 2010). 2010 kamen neue Paktpartner hinzu wie die Kultusministerkonferenz und die Beauftragte der Bundesregierung für Migration, Flüchtlinge und Integration 
(Busemeyer 2015a). Die am Pakt beteiligten Partner lobten regelmäßig den Erfolg des Instrumentes, während die Gewerkschaften weiterhin außen vor blieben. Die im Koalitionsvertrag wiederholt geäußerten Bekenntnisse zur Wichtigkeit des Konsensprinzips in der Berufsbildungspolitik deuteten darauf hin, dass sich diese untergeordnete Rolle der Gewerkschaften bei einer weiteren Neuauflage des Ausbildungspaktes verändern könnte.

So wurde dann tatsächlich im Dezember 2014 unter Einbeziehung der Gewerkschaften die neue „Allianz für Aus- und Weiterbildung“ ins Leben gerufen. In der Allianz wurden neben den Gewerkschaften auch weitere Partner neu aufgenommen, wie zum Beispiel die Arbeits- und Sozialministerkonferenz der Länder und die Bundesagentur für Arbeit (Allianz 2014). Die Ziele der Allianz setzen die bisherigen Schwerpunktsetzungen fort: Stärkung der beruflichen - insbesondere der betrieblichen - Bildung, Förderung der Gleichwertigkeit von beruflicher und akademischer Bildung, Verbesserung der Übergänge in den Ausbildungs- und Karrierepfaden von Jugendlichen, Verbesserung der Berufsberatung in Schulen sowie die Bekämpfung von „Matching“-Problemen auf dem Ausbildungsmarkt durch intensivere Vermittlung.

Im Unterschied zu den Vorgänger-Pakten enthält die Allianz verbindlichere Maßnahmen zur Lösung von Engpässen auf dem Ausbildungsstellenmarkt. So verpflichtete sich die Wirtschaft, im Jahr 201520.000 zusätzliche Ausbildungsplätze bereitzustellen und dieses Niveau auch in den Folgejahren zu halten (ebd.: 4). Hinzu kommen 500.000 Praktikumsplätze für Schüler*innen sowie eine Selbstverpflichtung, die einer Art Ausbildungsgarantie nahekommt: Jedem „vermittlungsbereiten“ Jugendlichen, der zum 30.9. noch keinen Ausbildungsplatz hat, macht „die Wirtschaft" drei Angebote für eine betriebliche Ausbildung - allerdings mit der Einschränkung, dass Jugendliche beruflich und räumlich mobil sind (ebd.: 4). Des Weiteren wird durch die Allianz ein neues Förderinstrument eingeführt: die "assistierte Ausbildung“. In der assistierten Ausbildung werden Berufsvorbereitung und Ausbildung verknüpft, indem Betrieben, die Jugendliche mit besonderem Förderbedarf ausbilden, gezielt und passgenau Unterstützungsmaßnahmen angeboten werden. Die Allianz sieht die Schaffung von zunächst 10.000 Plätzen in der assistierten Ausbildung vor. Das Instrument wird von den Paktpartnern - inklusive den Gewerkschaften - positiv bewertet (Busemeyer 2015a).

Abschließend sei noch die Einführung des Deutschen Qualifikationsrahmens (DQR) erwähnt. Dieser trat zwar noch vor Beginn der Großen Koalition in Kraft (im Mai 2013), wurde dann allerdings in der 18. Legislaturperiode weiterentwickelt, da nun schrittweise die bestehenden Qualifikationen den Niveaustufen des DQR zugeordnet werden mussten. Der DQR geht zurück auf die EU-Initiative zur Etablierung eines Europäischen Qualifikationsrahmens (EQR), der als Übersetzungs- 
instrument Transparenz und Vergleichbarkeit von Qualifikationen innerhalb der EU fördern soll. Im Zusammenhang mit der Einführung des DQR wurde in den vorangehenden Jahren die Einführung eines Europäischen Kreditpunktesystems im Bereich der beruflichen Bildung (ECVET - European Credit System for Vocational Education and Training) diskutiert. In Deutschland verlief diese Diskussion sehr kontrovers, da Gewerkschaften, aber auch das Handwerk, durch die mit ECVET in Verbindung gebrachte „Modularisierung“ eine Unterminierung des Berufsprinzips fürchteten (Busemeyer 2009a, Trampusch 2008).

Angesichts dieser Vorgeschichte ist es erstaunlich, dass die weitere Entwicklung des DQR und der deutschen Variante von ECVET (D-ECVET) vergleichsweise unkontrovers und weitestgehend von der Öffentlichkeit unbemerkt verlief. Der Beschluss des Arbeitskreises DQR, in dem alle relevanten bildungspolitischen Akteure von KMK, Bundesregierung, Hochschulen bis zu Gewerkschaften und Arbeitgebern vertreten waren, machte bereits deutlich, dass der DQR zwar die Transparenz und Vergleichbarkeit der Abschlüsse und Qualifikationen verbessern sollte, dabei aber „das bestehende System der Zugangsberechtigungen nicht ersetzt“ (AK DQR 2011: 5). In einer bemerkenswerten gemeinsamen Stellungnahme von BDA, DIHK, ZDH, DGB und Hochschulrektorenkonferenz (HRK) sprachen sich die Unterzeichner 2016 klar gegen jüngere Bemühungen der Europäischen Kommission aus, den EQR in Richtung eines echten „Berechtigungsinstruments“ weiterzuentwickeln (BDA et al. 2016). Der DQR habe sich als „Transparenzinstrument" bewährt, solle aber vom Anspruch her auch nicht darüber hinausgehen. Zur Einführung eines ECVET-Systems war in der vorangehenden Legislaturperiode eine Pilotinitiative gestartet worden (DECVET), die die Schaffung von Ausbildungsbausteinen am Beispiel ausgewählter Berufe erproben sollte. Auch wenn die DECVET-Initiative zunächst kontrovers diskutiert wurde, hat dies kaum Auswirkungen auf das deutsche Berufsbildungssystem gehabt. Die Anwendung von ECVET-Prinzipien erfolgt nach dem „bottom-up-Prinzip“ und ordnet sich dabei „nationalen und regionalen Bestimmungen unter" (DEQA-VET o. J.). Augenblicklich scheint es keinen Akteur zu geben, der sich entschieden für eine stärkere Modularisierung der Ausbildung einsetzt, so dass die ECVET-Initiative mehr oder weniger im Sande verläuft.

\subsection{Schulpolitik und frühkindliche Bildung}

Auch wenn die Wahlprogramme der Parteien zur Bundestagswahl 2013 durchaus schulpolitische Forderungen enthielten, wählt der Koalitionsvertrag in diesem Bereich eine sehr zurückhaltende Sprache und spricht lediglich abstrakt von der Förderung von kultureller, politischer sowie mathematisch-naturwissenschaftli- 
cher Bildung sowie gemeinsam mit den Ländern anzugehenden Bemühungen zur Digitalisierung von Schulen (CDU/CSU/SPD 2013: 22f.). Von 2003 bis 2009 hatte sich die Bundesregierung im Rahmen eines $4 \mathrm{Mrd}$. Euro schweren Investitionsprogramms am Ausbau von Ganztagsschulen beteiligt (Mahner/Wolf 2010). In der 18. Legislaturperiode beschränkte sich der Bund auf die forschungsgeleitete Begleitung und Evaluation des Ganztagsschulausbaus im Rahmen der Begleitforschung „Studie zur Entwicklung von Ganztagsschulen - StEG“. Weitere Schwerpunkte der Arbeit des Bundes in der Schulpolitik waren die Durchführung von internationalen Vergleichsstudien sowie die Förderung der empirischen Bildungsforschung und der Bildungsstatistik, wie in Art. 91b GG vorgesehen.

Ein stärkeres Engagement des Bundes ist im Feld der frühkindlichen Erziehung zu beobachten, allerdings stärker unter familien- als unter bildungspolitischen Vorzeichen (vgl. dazu auch das Kapitel von Henninger/von Wahl i.d.B.). Um die Länder und Kommunen bei der Umsetzung des seit dem 1. August 2013 bestehenden elterlichen Rechtsanspruchs auf Betreuung von Kindern ab dem ersten Lebensjahr zu entlasten, hat die Bundesregierung nach dem 1. Investitionsprogramm „Kinderbetreuungsfinanzierung" (2008-2013) inzwischen drei weitere aufgelegt. Das dritte und vierte Investitionsprogramm fielen in die Verantwortung der Großen Koalition, die damit den bereits unter den Vorgängerregierungen begonnenen Expansionspfad fortführte. Das aktuelle vierte Investitionsprogramm wurde im Juni 2017 beschlossen. In diesem Rahmen stellt der Bund den Ländern im Zeitraum von 2017 bis 2021 insgesamt 1,126 Mrd. Euro zur Finanzierung von 100.000 weiteren Betreuungsplätzen zur Verfügung. Der 2015 veröffentlichte Fünfte Bericht zur Evaluation des Kinderförderungsgesetzes dokumentiert einen massiven Anstieg der Zahl der Kinder unter drei Jahren in Tagesbetreuung von ca. $360.000 \mathrm{im} \mathrm{Jahr}$ 2008 auf 660.000 im Jahr 2014 (BMFSFJ 2015: 1), verbunden mit einem Anstieg des Anteils der Kleinkinder in Tagesbetreuung (gemessen an der Gesamtzahl der Kleinkinder im jeweiligen Alter) von etwa 17,6 Prozent auf 32,3 Prozent.

Die starke quantitative Expansionsdynamik weckte Bedenken hinsichtlich der Qualität der Kinderbetreuung. Auch die bestehenden Unterschiede zwischen Bundesländern im Ausbau des Betreuungsangebots sorgten für Unmut. Der Evaluationsbericht des Kinderförderungsgesetzes zeigt, dass die Beteiligungsquoten (und damit die „quantitative“ Versorgung) in den neuen Bundesländern weiterhin deutlich über den Quoten in den alten Bundesländern liegt. Allerdings sind in den neuen Bundesländern die Betreuungsrelationen ungünstiger (ebd.: 4): In Ostdeutschland (ohne Berlin) kommen 2014 statistisch betrachtet 5,8 Kinder auf eine Betreuungskraft (bei unter Drei-Jährigen), während es in Westdeutschland nur 3,4 Kinder sind. Die Bertelsmann Stiftung hatte bei einer Anhörung im Bundestag 2014 eine Betreuungsrelation von 1:3 für Kinder unter drei Jahren und von 
1:7,5 für 3-6-jährige Kinder empfohlen (Bertelsmann Stiftung 2014). Die Stiftung kritisierte darüber hinaus die Tatsache, dass bei der Berechnung der offiziellen Betreuungsrelation zu wenig die Tatsache berücksichtigt werde, dass das Personal neben der reinen Betreuung auch anderen Aufgaben wie Fortbildung, Dokumentation, Gespräche mit Kolleg^innen und Eltern übernehme. Insgesamt müsste somit das Betreuungspersonal massiv aufgestockt werden, um eine hochqualitative Betreuung zu ermöglichen.

Die Bundesregierung leitete zusammen mit den Ländern im November 2014 einen politischen Diskussions- und Abstimmungsprozess ein, der gesetzliche Maßnahmen zur Verbesserung der Qualität der Kinderbetreuung entwickeln sollte (BMFSFJ, JFMK 2014). Hiermit wurde unmittelbar an eine Vorgabe des Koalitionsvertrags angeknüpft (siehe oben). Ein zentraler Diskussionspunkt in diesem gut zweijährigen „Qualitäts-Prozess“ war die Frage, ob bundeseinheitliche Qualitätsstandards gesetzlich geregelt werden sollten oder nicht. Die Wohlfahrtsverbände und die Gewerkschaften sprachen sich gemeinsam klar für eine bundeseinheitliche Regelung von Qualitätsstandards aus (AWO/Caritas/GEW 2014), während die kommunalen Spitzenverbände auf die Erhaltung lokaler Autonomie drängten (Deutscher Städtetag 2014).

Im Mai 2017 wurden - noch unter Führung von Familienministerin Manuela Schwesig (SPD) - von Bund und Ländern gemeinsame „Eckpunkte für bessere Qualität in der Kindertagesbetreuung" beschlossen (BMFSFJ 2017). Diese Eckpunkte sehen im Wesentlichen die Verabschiedung eines Qualitätsentwicklungsgesetzes vor. Im Rahmen dieses für die Zukunft geplanten Gesetzes werden den Ländern Bundesmittel zur Verfügung gestellt, die sie zur Verbesserung der Qualität der Kindertagesbetreuung verwenden. Sie können dabei flexibel aus einer Reihe von Qualitätsmaßnahmen auswählen, sind aber dem Bund in Form von individuellen Zielvereinbarungen Rechenschaft schuldig. Eine breite Allianz aus 30 Wohlfahrts-, Familien- und Kinderrechtsverbänden, Gewerkschaften und Stiftungen begrüßten die prinzipielle Entscheidung zur Einführung einheitlicher Qualitätsstandards für Kitas (AGF 2017). DGB und BDA forderten gar ein dauerhaftes Engagement des Bundes zur Sicherung der Qualität in Kitas (DGB/BDA 2017). Insofern zeigen die Zeichen eindeutig in Richtung eines fortdauernden Engagements des Bundes nicht nur in der Finanzierung, sondern zunehmend auch der Qualitätssicherung im Bereich der frühkindlichen Erziehung. 


\section{$5 \quad$ Fazit und Diskussion}

Die Bilanz der zweiten Großen Koalition unter Merkels Führung im Bereich Bildungspolitik fällt in der Gesamtbetrachtung eher positiv aus - wenn man die prinzipiellen politischen und rechtlichen Beschränkungen des deutschen Bildungsföderalismus als gegeben betrachtet. Die im Koalitionsvertrag angekündigten Initiativen ließen eher Kontinuität als radikalen Wandel erwarten. Dies lag allerdings auch daran, dass die strittige Frage der Abschaffung des Kooperationsverbots und der damit verbundenen Verlagerung der BAföG-Finanzierung auf den Bund im Vertrag nicht erwähnt wurde, obwohl diese Entscheidung die Dynamik der Bund-Länder-Beziehungen in der Hochschulpolitik langfristig prägen und verändern wird. Man mag die Tatsache kritisieren, dass die Abschaffung des Kooperationsverbots in der Hochschulpolitik lediglich die negativen Auswirkungen der Föderalismusreform ausglich, die eine andere Große Koalition gerade einmal fünf Jahre vorher beschlossen hatte. Auf der anderen Seite könnte man die Lernfähigkeit bildungspolitischer Akteure loben, die - trotz ideologisch unterschiedlicher Positionen - in pragmatischer und konsensorientierter Weise eine weitreichende Verfassungsreform beschlossen haben, die sich langfristig positiv auf deutsche Hochschulen und Forschungseinrichtungen auswirken dürfte. Es ist zu erwarten, dass sich der Bund auch über die Exzellenzstrategie hinaus in den nächsten Jahren wieder stärker in der Hochschulpolitik engagieren wird.

In der beruflichen Bildung war der Handlungsdruck wesentlich geringer als in der Hochschulpolitik, da weithin ein breiter Konsens über die Leistungsfähigkeit des deutschen Berufsbildungssystems herrscht. Auch wenn die Gewerkschaften in regelmäßigen Abständen auf Schwierigkeiten bei der Qualität einiger Ausbildungsberufe und bei Übergängen von Jugendlichen mit schwachen Schulqualifikationen hinweisen, ist dank der guten Lage auf dem Ausbildungs- und Arbeitsmarkt offensichtlich der Leidensdruck gegenwärtig nicht groß genug, um grundsätzliche Reformen des Systems anzustoßen. Durch die Weiterentwicklung der Ausbildungspakte zur "Allianz für Aus- und Weiterbildung" hat die Große Koalition zudem die Rolle der Gewerkschaften als gleichberechtigte Partner im korporatistischen Abstimmungsprozess wieder aufgewertet, nachdem es in den Jahren zuvor wiederholt zu einer gewissen Frontstellung zwischen den Gewerkschaften einerseits und der Bundesregierung im Verbund mit den Arbeitgebern andererseits gekommen war.

Im Bereich der frühkindlichen Erziehung hat die Große Koalition die Expansionspolitik der Vorgängerregierungen entschieden fortgesetzt und dabei - wie im Koalitionsvertrag angekündigt - zunehmend stärker auf Qualitätsaspekte in der Kinderbetreuung Wert gelegt. Sicherlich kann kritisiert werden, dass zum Ende der Legislaturperiode in dieser Frage lediglich ein Eckpunkte-Beschluss und noch kein 
Gesetzesbeschluss vorliegt. Allerdings ist es der Ministerin Schwesig gelungen, einen breiten Konsens hinsichtlich der Notwendigkeit einer auf Langfristigkeit angelegten Beteiligung des Bundes zu erzielen, was angesichts der Konfliktträchtigkeit der Auseinandersetzungen zwischen Bund und Ländern in der Schulpolitik erstaunt.

Alles in allem hat die Große Koalition somit ihre selbst gesteckten bildungspolitischen Hausaufgaben zufriedenstellend erledigt. $\mathrm{Zu}$ dieser prinzipiell positiven Einschätzung sei jedoch hinzuzufügen, dass die Beschränkungen des Bildungsföderalismus die Messlatte vergleichsweise niedrig setzen. Dies gilt insbesondere für die Schulpolitik. Der bereits erwähnte „Flickenteppich“ (Welsh 2015: 484) der Bundesländer hinsichtlich Schulstrukturen und Lehrerausbildung erschwert die Mobilität von Schüler*innen und Lehrer*innen und beeinträchtigt die Transparenz und Vergleichbarkeit von Bildungsabschlüssen - trotz anhaltender Bemühungen zur Schaffung von nationalen Bildungsstandards. Das Berufsbildungssystem und der Übergangssektor zwischen Schulbildung und Ausbildung sind weiterhin institutionell fragmentiert. Die Übergänge zwischen beruflicher und hochschulischer Bildung sowie zwischen unterschiedlichen Schultypen im Sekundarschulbereich sind immer noch schwieriger als in anderen Ländern und sorgen für Bildungsungleich heit. Die Grundfinanzierung der Hochschulen - besonders in finanzschwachen Bundesländern - ist weiterhin unzureichend, wenn sie nicht in den Genuss von Sondermitteln kommen. Inwiefern die im Herbst 2017 neu gewählte Bundesregierung diese tiefer liegenden Problematiken angehen wird, wird sich zeigen.

\section{Literatur}

$A K D Q R$, 2011: Deutscher Qualifikationsrahmen für lebenslanges Lernen, verabschiedet vom Arbeitskreis Deutscher Qualifikationsrahmen (AK DQR), 22.03.2011. Bonn/Berlin. Allianz für Aus- und Weiterbildung, 2014: Allianz für Aus- und Weiterbildung 2014-2018. Berlin.

Arbeitsgemeinschaft der deutschen Familienorganisationen e. V. (AGF), 2017: Gemeinsame Erklärung: Einheitliche Qualitätsstandards für Kitas, 02.06.2017, abrufbar unter: http:// www.ag-familie.de/media/docs17/170602_verbaendeerklaerung_Kitaqualitaet_ohne_teaser.pdf (01.09.2017).

AWO/Caritas/GEW, 2014: Bundespressekonferenz, Erklärung von Arbeiterwohlfahrt (Bundesverband), Deutscher Caritasverband, Gewerkschaft Erziehung und Wissenschaft (Hauptvorstand): Deutschland braucht ein Bundesqualitätsgesetz für die Kindertagesbetreuung. Berlin.

BDA/DIHK/ZDH/DGB/RK, 2016: DQR muss Transparenzinstrument bleiben: Gemeinsame Erklärung der Spitzenverbände der Deutschen Wirtschaft, des Deutschen Gewerkschafts- 
bundes sowie der Hochschulrektorenkonferenz für die berufliche und die hochschulische Bildung. Berlin.

Bertelsmann Stiftung, 2014: Öffentliche Anhörung zur Verbesserung des Ausbaus und der Qualität der Kindertagesstätten vom Ausschuss für Familie, Senioren, Frauen und Jugend. Deutscher Bundestag, Stellungnahme Kathrin Bock-Famulla, 10.11.2014. Berlin.

BMFSFJ/JFMK, 2014: Communiqué: Frühe Bildung weiterentwickeln und finanziell sichern. Berlin.

Bundesministerium der Finanzen (BMF), 2014: Finanzierung prioritärer Maßnahmen im Bildungsbereich steht, 27.05.2014, abrufbar unter: http://www.bundesfinanzministerium. de/Content/DE/Standardartikel/Themen/Oeffentliche_Finanzen/Foederale_Finanzbeziehungen/Laenderhaushalte/2014-05-27-Vorschlag-Verteilung-Mittel.html (31.08.2017).

Bundesministerium für Bildung und Forschung (BMBF), 2016a: Gesamtpaket für die Hochschulen beschlossen, 17.06.2016, abrufbar unter: https://www.bmbf.de/de/gesamtpaket-fuer-die-hochschulen-beschlossen-3017.html (31.08.2017),

Bundesministerium für Bildung und Forschung (BMBF), 2016b: Evaluation des Berufsbildungsgesetzes (BBiG): Evaluierungsbericht. Bonn/Berlin.

Bundesministerium für Bildung und Forschung (BMBF), o. J. a: Pakt für Forschung und Innovation, abrufbar unter: https://www.bmbf.de/de/pakt-fuer-forschung-und-innovation-546.html (31.08.2017).

Bundesministerium für Bildung und Forschung (BMBF), o. J. b: Wissenschaftszeitvertragsgesetz, abrufbar unter: https://www.bmbf.de/de/karrierewege-fuer-den-wissenschaftlichen-nachwuchs-an-hochschulen-verbessern-1935.html (31.08.2017).

Bundesministerium für Familien, Senioren, Frauen und Jugend (BMFSFJ), 2015: Fünfter Bericht zur Evaluation des Kinderförderungsgesetzes, Kurzfassung. Berlin.

Bundesministerium für Familien, Senioren, Frauen und Jugend (BMFSFJ), 2017: Eckpunkte für bessere Qualität in der Kindertagesbetreuung beschlossen, Pressemitteilung 19.05.2017, abrufbar unter: https://www.bmfsfj.de/bmfsff/aktuelles/alle-meldungen/eckpunkte-fuer-bessere-qualitaet-in-der-kindertagesbetreuung-beschlossen/116338 (01.09.2017).

Bundesregierung, 2016: Fördersätze für Meister-BAföG steigen, Pressemitteilung 18.03.2016, abrufbar unter: https://www.bundesregierung.de/Content/DE/Artikel/2015/10/2015-10-14-dritte-novelle-meister-bafoeg.html (01.09.2017).

Bündnis 90/Die Grünen, 2013: Zeit für den grünen Wandel: Bundestagswahlprogramm 2013 von BÜNDNIS 90/DIE GRÜNEN. Berlin.

Busemeyer, Marius R., 2007: The Determinants of Public Education Spending in 21 OECD Democracies, 1980-2001, in: Journal of European Public Policy 14: 582-610.

Busemeyer, Marius R., 2009a: Die Europäisierung der deutschen Berufsbildungspolitik: Sachzwang oder Interessenpolitik? Friedrich-Ebert-Stiftung, Abt. Internationale Politikanalyse. Bonn.

Busemeyer, Marius R., 2009b: Wandel trotz Reformstau: Die Politik der beruflichen Bildung seit 1970. Frankfurt/New York.

Busemeyer, Marius R., 2012: Business as a Pivotal Actor in the Politics of Training Reform: Insights from the Case of Germany, in: British Journal of Industrial Relations 50: 690-713.

Busemeyer, Marius R., 2015a: Aufbruch oder Stillstand in der Berufsbildungspolitik? Die neue Allianz für Aus- und Weiterbildung, WISO direkt, Juli 2015.

Busemeyer, Marius R., 2015b: Skills and Inequality: The Political Economy of Education and Training Reforms in Western Welfare States. Cambridge/New York. 
Busemeyer, Marius R./Franzmann, Simon T./Garritzmann, Julian L., 2013: Who Owns Education? Cleavage Structures in the Partisan Composition over Educational Expansion, in: West European Politics 36: 521-546.

Busemeyer, Marius R./Garritzmann, Julian L./Neimanns, Erik/Nezi, Roula, 2018: Investing in Education in Europe: Evidence from a Survey of Public Opinion, in: Journal of European Social Policy 28: 34-54.

Busemeyer, Marius R./Seitzl, Lina, 2017: The Partisan Politics of Early Childhood Education in the German Länder, in: Journal of Public Policy (FirstView), abrufbar unter: https:// doi.org/10.1017/S0143814X16000313.

Castles, Francis G., 1989: Explaining Public Education Expenditure in OECD Nations, in: European Journal of Political Research 17: 431-448.

Castles, Francis G., 2013: The Real Issue for Future Comparative Policy Research: Does Government Matter?, in: Armingeon, Klaus (Hrsg.): Staatstätigkeiten, Parteien und Demokratie: Festschrift für Manfred G. Schmidt. Wiesbaden, 185-204.

CDU/CSU, 2013: Gemeinsam erfolgreich für Deutschland: Regierungsprogramm 2013-2017. Berlin/München.

CDU/CSU/SPD, 2013: Deutschlands Zukunft gestalten: Koalitionsvertrag zwischen CDU, CSU und SPD. Berlin.

Demling, Alexander, 2014: Diese Bundesländer sparen am meisten an ihren Hochschulen, 08.10.2014, abrufbar unter: http://www.spiegel.de/lebenundlernen/uni/hochschulen-diese-unis-sparen-am-meisten-a-995977.html (31.08.2017).

Deutsche Referenzstelle für Qualitätssicherung in der beruflichen Bildung (DEQA-VET), o. J.: ECVET: Europäisches Leistungspunktesystem für die Berufsbildung, abrufbar unter: https://www.deqa-vet.de/de/ECVET-2258.php (01.09.2017).

Deutscher Bundestag, 2017: Stellungnahme Öffentliches Fachgespräch zum Thema „Berufliche Bildung - einschließlich BBiG“, Bundesvereinigung Deutscher Arbeitgeberverbände (BDA), Barbara Dorn. Berlin.

Deutscher Städtetag, 2014: Kommunen wollen weitere Schritte für gute Betreuungsqualität, Pressemitteilung 06.11.2014. Berlin.

$D G B, 2016$ : DGB Einschätzung des Evaluationsberichts zum BBiG. Berlin.

$D G B / B D A$, 2017: DGB und BDA fordern dauerhafte Bundesbeteiligung für mehr Qualität an Kitas, Pressemitteilung 22.06.2017, abrufbar unter: https://www.arbeitgeber.de/www/ arbeitgeber.nsf/res/PI02217.pdf/\$file/PI02217.pdf (01.09.2017).

Die Linke, 2013: 100 \% Sozial: Wahlprogramm zur Bundestagswahl 2013. Berlin.

FDP, 2013: Bürgerprogramm 2013: Damit Deutschland stark bleibt. Berlin.

Fleckenstein, Timo/Saunders, Adam M./Seeleib-Kaiser, Martin, 2011: The Dual Transformation of Social Protection and Human Capital: Comparing Britain and Germany, in: Comparative Political Studies 44: 1622-1650.

Helbig, Marcel/Nikolai, Rita, 2015: Die Unvergleichbaren: Der Wandel der Schulsysteme in den deutschen Bundesländern seit 1949. Bad Heilbrunn.

Henninger, Annette/von Wahl, Angelika, 2015: Drei Schritte vor und zwei zurück? Familien- und Gleichstellungspolitik 2009-2013, in: Zohlnhöfer, Reimut/Saalfeld, Thomas (Hrsg.): Politik im Schatten der Krise: Eine Bilanz der Regierung Merkel 2009-2013. Wiesbaden, 451-468.

Hepp, Gerd F., 2011: Bildungspolitik in Deutschland: Eine Einführung. Wiesbaden. 
Himmelrath, Armin, 2014: Das Kooperationsverbot muss weg: Vorstoß aus Niedersachsen, 02.09.2014, abrufbar unter: http://www.spiegel.de/lebenundlernen/schule/kooperationsverbot-niedersachsen-fordert-korrektur-ueber-bundesrat-a-989467.html (06.03.2018).

Jakobi, Anja P., 2011: Political Parties and the Institutionalization of Education: A Comparative Analysis of Party Manifestos, in: Comparative Education Review 55: 189-209.

Konsortium Bundesbericht Wissenschaftlicher Nachwuchs, 2017: Bundesbericht Wissenschaftlicher Nachwuchs 2017. Statistische Daten und Forschungsbefunde zur Promovierenden und Promovierten in Deutschland. Bielefeld.

Kramer, Bernd, 2015: Uni-Prekariat: Lobby will Befristung für alle, 10.06.2015, abrufbar unter: http://www.spiegel.de/lebenundlernen/uni/hochschulen-wehren-sich-gegen-strenge-befristungsregeln-a-1037865.html (06.03.2018).

Lehmbruch, Gerhard, 2000: Parteienwettbewerb im Bundesstaat: Regelsysteme und Spannungslagen im politischen System der Bundesrepublik Deutschland. Wiesbaden.

Mahner, Sebastian/Wolf, Frieder, 2010: Die Bildungspolitik der Großen Koalition, in: Egle, Christoph/Zohlnhöfer, Reimut (Hrsg.): Die zweite Große Koalition: Eine Bilanz der Regierung Merkel 2005-2009. Wiesbaden, 380-402.

Niemann, Dennis, 2010: Deutschland - Im Zentrum des PISA-Sturms, in: Knodel, Philipp/ Martens, Kerstin/de Olano, Daniel/Popp, Marie (Hrsg.): Das PISA-Echo: Internationale Reaktionen auf die Bildungsstudie. Frankfurt/New York, 59-90.

Rauh, Christian/Kirchner, Antje/Kappe, Roland, 2011: Political Parties and Higher Education Spending: Who Favours Redistribution? in: West European Politics 34: 1185-1206.

Schmidt, Manfred G., 1980: CDU und SPD an der Regierung: Ein Vergleich ihrer Politik in den Ländern. Frankfurt/New York.

Schmidt, Manfred G., 2002: Warum Mittelmaß? Deutschlands Bildungsausgaben im internationalen Vergleich, in: Politische Vierteljahresschrift 43: 3-19.

Schmidt, Manfred G., 2007a: Warum nicht einmal Mittelmaß? Die Finanzierung der deutschen Hochschulen im internationalen Vergleich, in: Gesellschaft - Wirtschaft - Politik 56: $465-480$.

Schmidt, Manfred G., 2007b: Testing the Retrenchment Hypothesis: Educational Spending, 1960-2002, in: Castles, Francis G. (Hrsg.): The Disappearing State? Retrenchment Realities in an Age of Globalisation. Cheltenham/Northampton, 159-183.

Schmidt, Manfred G., 2008: Germany: The Grand Coalition State, in: Colomer, Josep M. (Hrsg.): Comparative European Politics. Milton Park/New York, 58-93.

Schmoll, Heike, 2015: Von der Hand in den Mund: Verwendung von Bafög-Mitteln, 07.04.2015, abrufbar unter: http://www.faz.net/aktuell/politik/inland/johanna-wanka-will-bafoeg-entlastung-in-hochschulen-stecken-13525680/studierende-wie-hier-an-der-13525798.html (06.03.2018).

Solga, Heike, 2014: Education, Economic Inequality and the Promises of the Social Investment State, in: Socio-Economic Review 12: 269-297.

SPD, 2013: Das WIR entscheidet: Das Regierungsprogramm 2013-2017. Berlin.

Spiegel Online, 2016: Nachfolger der Exzellenzinitiative abgesegnet, 16.06.2016, abrufbar unter: http://www.spiegel.de/lebenundlernen/uni/exzellenzinitiative-milliardenprogramm-fuer-elite-unis-abgesegnet-a-1098099.html (31.08.2017).

Spiegel Online, 2017: Prekär ist immer noch normal, 17.03.2017, abrufbar unter: http://www. spiegel.de/lebenundlernen/uni/befristete-jobs-an-hochschulen-ein-jahr-wissenschaftszeitvertragsgesetz-wisszeitvg-a-1139090.html (31.08.2017). 
Spiewak, Martin, 2013: Die selbstverordnete Impotenz in der Schulpolitik, 12.11.2013, abrufbar unter: http://www.zeit.de/gesellschaft/schule/2013-11/koalitionsverhandlungen-kooperationsverbot-bildungspolitik (06.03.2018).

Statista, 2018: Anzahl der Studierenden an Hochschulen in Deutschland vom Wintersemester 2002/2003 bis 2017/2018, abrufbar unter: https:/de.statista.com/statistik/daten/ studie/221/umfrage/anzahl-der-studenten-an-deutschen-hochschulen/ (06.03.2018).

Statistisches Bundesamt, 2016: Bildungsfinanzbericht 2016. Wiesbaden.

Streeck, Wolfgang/Thelen, Kathleen, 2005: Introduction: Institutional Change in Advanced Political Economies, in: Streeck, Wolfgang/Thelen, Kathleen (Hrsg.): Beyond Continuity: Institutional Change in Advanced Political Economies. Oxford/New York, 1-39.

Titz, Christoph, 2014: Wankas Visionen: Vorschlag zur Grundgesetzänderung, 17.06.2014, abrufbar unter: http://www.spiegel.de/lebenundlernen/uni/grundgesetz-wanka-will-kooperationsverbot-bei-hochschulen-aufweichen-a-975702.html (06.03.2018).

Trampusch, Christine, 2008: Jenseits von Anpassungsdruck und Lernen: die Europäisierung der deutschen Berufsbildung, in: Zeitschrift für Staats- und Europawissenschaften 6: 577-605.

Welsh, Helga A., 2015: Kontinuität als Markenzeichen: Die Bildungspolitik der christlich-liberalen Koalition (2009-2013), in: Zohlnhöfer, Reimut/Saalfeld, Thomas (Hrsg.): Politik im Schatten der Krise. Eine Bilanz der Regierung Merkel 2009-2013. Wiesbaden, 469-491.

Wiarda, Jan-Martin, 2015: Exzellenzinitiative: Wohin mit den Elite-Milliarden?, 07.10.2015, abrufbar unter: http://www.spiegel.de/lebenundlernen/uni/exzellenzinitiative-milliarden-zu-verteilen-nur-wie-a-1056589.html (06.03.2018).

Wolf, Frieder, 2006: Die Bildungsausgaben der Bundesländer im Vergleich: Welche Faktoren erklären ihre beträchtliche Variation? Münster. 